\title{
ON TRANSLATION INVARIANT MULTIRESOLUTION ANALYSIS
}

\author{
Angel San Antolín \\ University of Alicante, Spain
}

\begin{abstract}
We give a characterization of the scaling functions and low pass filters in a translation invariant multiresolution analysis on $L^{2}\left(\mathbb{R}^{n}\right)$. Our conditions involve the notion of locally non-zero function. We write our results in a general context where one considers a dilation given by a fixed expansive linear map on $\mathbb{R}^{n}$ preserving the integer lattice $\mathbb{Z}^{n}$. Indeed, for any such a linear map we construct a scaling function where the support of the Fourier transform is bounded and does not contain any open neighborhood of the origin.
\end{abstract}

\section{INTRODUCTION}

Let $A: \mathbb{R}^{n} \rightarrow \mathbb{R}^{n}, n \geq 1$, be an expansive linear map such that $A \mathbb{Z}^{n} \subset \mathbb{Z}^{n}$. A linear map $A$ is said to be expansive if all (complex) eigenvalues of $A$ have absolute value greater than 1 . Here and further we use the same notation for the linear map $A$ and its corresponding matrix with respect to the canonical base.

In this paper, we write a characterization of the orthonormal scaling functions $\phi \in L^{2}\left(\mathbb{R}^{n}\right)$ such that $|\widehat{\phi}|=\chi_{S}$, where $\widehat{\phi}$ means the Fourier transform of $\phi$. Our starting point is the main result in [5]. Using the notion of locally non-zero function, we achieve more understanding on the behavior of $\widehat{\phi}$ in a neighborhood of the origin. It is known the existence of scaling functions in a multiresolution analysis defined in $L^{2}(\mathbb{R})$ and with dyadic dilation such that the origin is not a continuity point for their Fourier

2010 Mathematics Subject Classification. 42C40, 42C30, 51M15.

Key words and phrases. Fourier transform, locally nonzero function, low pass filter, scaling function, translation invariant multiresolution analysis. (Spain).

This work was partially supported by MEC/MICINN grant \#MTM2011-27998 
transform. Sometimes, these are considered as pathological cases of scaling functions. Here, our characterization let us construct a bounded measurable set $G \subset \mathbb{R}^{n}$ such that $G$ and $\mathbb{R}^{n} \backslash G$ do not contain any open neighborhood of the origin and also the function $\theta \in L^{2}\left(\mathbb{R}^{n}\right)$ defined by $\widehat{\theta}=\chi_{G}$ is an orthonormal scaling function in a multiresolution analysis associated to the dilation $A$.

Moreover, if we have a scaling function $\phi \in L^{2}\left(\mathbb{R}^{n}\right)$ such that $|\widehat{\phi}|=$ $\chi_{S}$ in a multiresolution analysis associated to the dilation $A$, we will see that its associated low pass filter $H$ is $\mathbb{Z}^{n}$-periodic and such that $|H|=\chi_{E}$ for a measurable set $E \subset \mathbb{R}^{n}$. We characterize those low pass filters in a multiresolution analysis associated to the dilation $A$. A first approach to characterize those low pass filters in a multiresolution analysis with the dyadic dilation and $n=1$ was presented in the paper by E. Hernández, X. Wang and G. Weiss [15]. They assume that $E$ contains a neighborhood of the origin. Here we do not use any extra assumptions on the regularity at the origin of $\chi_{E}$.

A multiresolution analysis (MRA) is a general method introduced by Mallat [21] and Meyer [22] for constructing wavelets. Afterwards, the concept of MRA was considered on $L^{2}\left(\mathbb{R}^{n}\right), n \geq 1$, (see $\left.[11,20,28,30]\right)$ in a more general context where instead of the dyadic dilation one considers the dilation given by a fixed expansive linear map $A$ preserving the integer lattice. Given such a linear map $A$ one defines an $A$-MRA as a sequence of closed subspaces $V_{j}$, $j \in \mathbb{Z}$, of the Hilbert space $L^{2}\left(\mathbb{R}^{n}\right)$ that satisfies the following conditions:

(i) for any $j \in \mathbb{Z}, \quad V_{j} \subset V_{j+1}$;

(ii) for any $j \in \mathbb{Z}, \quad f(\mathbf{x}) \in V_{j} \Leftrightarrow f(A \mathbf{x}) \in V_{j+1}$;

(iii) $\overline{\cup_{j \in \mathbb{Z}} V_{j}}=L^{2}\left(\mathbb{R}^{n}\right)$;

(iv) There exists a function $\phi \in V_{0}$, that is called scaling function, such that $\left\{\phi(\mathbf{x}-\mathbf{k}): \mathbf{k} \in \mathbb{Z}^{n}\right\}$ is an orthonormal basis for $V_{0}$.

One of the possible ways for constructing a multiresolution analysis is to start with a scaling function $\phi \in L^{2}\left(\mathbb{R}^{n}\right)$. Beginning from Mallat's work [21], several authors have studied properties of the scaling functions. Let us suggest the paper by Soto-Bajo [27] where a survey on previous results of characterizations of scaling functions appears.

A key tool to work with scaling functions is the Fourier transform. We adopt the convention that the Fourier transform of a function $f \in L^{1}\left(\mathbb{R}^{n}\right) \cap$ $L^{2}\left(\mathbb{R}^{n}\right)$ is defined by

$$
\widehat{f}(\mathbf{y})=\int_{\mathbb{R}^{n}} f(\mathbf{x}) e^{-2 \pi i \mathbf{x} \cdot \mathbf{y}} d \mathbf{x}
$$

where $\mathbf{x} \cdot \mathbf{y}$ means the inner product in $\mathbb{R}^{n}$ of $\mathbf{x}$ and $\mathbf{y}$.

If $\phi$ is a scaling function of an $A$-MRA, then $d_{A}^{-1} \phi\left(A^{-1} \mathbf{x}\right) \in V_{-1} \subset V_{0}$ where $d_{A}=|\operatorname{det} A|$. By the condition (iv) we express this function in terms 
of the orthonormal basis $\left\{\phi(\mathbf{x}-\mathbf{k}): \mathbf{k} \in \mathbb{Z}^{n}\right\}$ as

$$
d_{A}^{-1} \phi\left(A^{-1} \mathbf{x}\right)=\sum_{\mathbf{k} \in \mathbb{Z}^{n}} a_{\mathbf{k}} \phi(\mathbf{x}-\mathbf{k}),
$$

where the convergence is in $L^{2}\left(\mathbb{R}^{n}\right)$ and $\sum_{\mathbf{k} \in \mathbb{Z}^{n}}\left|a_{\mathbf{k}}\right|^{2}<\infty$. Taking the Fourier transform we obtain

$$
\widehat{\phi}\left(A^{*} \mathbf{t}\right)=H(\mathbf{t}) \widehat{\phi}(\mathbf{t}) \quad \text { a.e. on } \mathbb{R}^{n},
$$

where $A^{*}$ is the adjoint map of $A$ and

$$
H(\mathbf{t})=\sum_{\mathbf{k} \in \mathbb{Z}^{n}} a_{\mathbf{k}} e^{-2 \pi i \mathbf{k} \cdot \mathbf{t}}
$$

is a $\mathbb{Z}^{n}$-periodic function which is called low pass filter associated to the scaling function $\phi$ in an $A$-MRA, or shortly low pass filter in an A-MRA. We will see a discussion on previous results on low pass filters at the end of Section 3.

In this paper, we focus on the notion of translation invariant $A$-MRA. It was introduced by W. R. Madych ([20]).

Definition 1.1. Let $\left\{V_{j}\right\}_{j \in \mathbb{Z}}$ be an $A$-MRA. If for any $f \in V_{0}$ and $\mathbf{u} \in \mathbb{R}^{n}$ we have $f(\mathbf{x}+\mathbf{u}) \in V_{0}$, then $\left\{V_{j}\right\}_{j \in \mathbb{Z}}$ is called a translation invariant $A$-MRA.

A classical example of a scaling function in a translation invariant multiresolution analysis defined on $L^{2}(\mathbb{R})$ with the dyadic dilation is the well known Shannon scaling function.

The following characterization of the scaling functions in a translation invariant $A$-MRA was proved by W. R. Madych ([20]).

Theorem A. Let $\phi \in L^{2}\left(\mathbb{R}^{n}\right)$. Then the function $\phi$ generates a translation invariant $A-M R A$ if and only if $|\widehat{\phi}|=\chi_{S}$ where $\chi_{S}$ is the characteristic function of the measurable set $S \subset \mathbb{R}^{n}$ which has the following properties:

(A) $S \subset A^{*} S$ (except a null measurable set);

(B) $S \cap\{S+\mathbf{k}\} \simeq \emptyset$ for any element $\mathbf{k}$ in $\mathbb{Z}^{n} \backslash\{\mathbf{0}\}$;

(C)

$$
\bigcup_{\mathbf{k} \in \mathbb{Z}^{n}}\{S+\mathbf{k}\} \simeq \mathbb{R}^{n}
$$

(D)

$$
\lim _{j \rightarrow \infty} \frac{1}{\left|A^{*-j} Q\right|} \int_{A^{*-j} Q} \chi_{S}(\mathbf{t}) d \mathbf{t}=1
$$

for every cube $Q$ of finite diameter in $\mathbb{R}^{n}$ where $A^{*}$ is the adjoint of $A$.

In addition, the condition $(\mathbf{D})$ can be replaced by any one of the following properties was observed in [20]:

- $\bigcup_{j \in \mathbb{Z}} L^{2}\left(A^{* j} S\right)$ is dense in $L^{2}\left(\mathbb{R}^{n}\right)$; 
- $\bigcup_{j \in \mathbb{Z}}\left(A^{* j} S\right) \simeq \mathbb{R}^{n}$.

The scaling functions $\phi$ such that $\widehat{\phi}=\chi_{S}$ are sometime called Minimally Supported Frequency (MSF) scaling functions. A characterization of the measurable sets $S \subset \mathbb{R}$ for which a function $\phi \in L^{2}(\mathbb{R})$ such that $|\widehat{\phi}|=\chi_{S}$ is a scaling function in a classical multiresolution analysis was proved by $\mathrm{M}$. Papadakis ([23]) independently of W. R. Madych's work. Afterwards, M. Papadakis, H. Sikić and G. Weiss ([24]) observed that one condition obtained in [23] is redundant. Moreover, they construct examples of such bounded measurable sets $S$. In the paper by M. Bownik, Z. Rzeszotnik and D. Speegle ([3]), other necessary and sufficient conditions on measurable sets $S \subset \mathbb{R}^{n}$ for which a function $\phi \in L^{2}\left(\mathbb{R}^{n}\right)$ such that $|\widehat{\phi}|=\chi_{S}$ is a scaling function of an $A$-MRA have been proved. The same question for some subspaces of $L^{2}\left(\mathbb{R}^{n}\right)$ was addressed in a joint work with K. S. Kazarian ([17]). Furthermore, a characterization of the functions $\phi \in L^{2}\left(\mathbb{R}^{n}\right)$ such that $|\widehat{\phi}|$ is a characteristic function and it generates a Parseval Frame $A$-MRA was proved by Bakić and Wilson ([1]). A set $S \subset \mathbb{R}^{n}$ as above was constructed by Q. Gu and D. Han ([12]) assuming $d_{A}=2$, and afterward, by M. Bownik, Z. Rzeszotnik and D. Speegle ([3]) without that additional hypothesis. An abstract relationship between translation invariant MRA and Minimally Supported Frequency wavelets is presented in $L^{2}(\mathbb{R})$ with dyadic dilation in the paper by Weber $([29])$.

Let us introduce some notation and definitions before formulating the results. $\mathbb{T}^{n}=\mathbb{R}^{n} / \mathbb{Z}^{n}$ and with some abuse of the notation we consider also that $\mathbb{T}^{n}$ is the unit cube $[0,1)^{n}$. If we write $f \in L^{2}\left(\mathbb{T}^{n}\right)$ we will understand that $f$ is defined on the whole space $\mathbb{R}^{n}$ as a $\mathbb{Z}^{n}$-periodic function.

We will denote $B_{r}(\mathbf{y})=\left\{\mathbf{x} \in \mathbb{R}^{n}:|\mathbf{x}-\mathbf{y}|<r\right\}$ and will write $B_{r}$ if $\mathbf{y}$ is the origin. For a set $E \subset \mathbb{R}^{n}$ we will denote $E^{c}=\mathbb{R}^{n} \backslash E$, the closure of the set $E$ by $\bar{E}$ and $A(E)=\left\{\mathbf{x} \in \mathbb{R}^{n}: \mathbf{x}=A \mathbf{t}\right.$ for $\left.\mathbf{t} \in E\right\}$. If $\mathbf{x} \in \mathbb{R}^{n}$ then we will write $\mathbf{x}+E=\{\mathbf{x}+\mathbf{y}$ : for $\mathbf{y} \in E\}$. Further, $E_{\mathbb{T}^{n}}=\left(E+\mathbb{Z}^{n}\right) \cap[0,1)^{n}$.

We say that $E \subset \mathbb{R}^{n}$ is a measurable set if it is Lebesgue measurable. Its Lebesgue measure will be denoted by $|E|_{n}$. If we do not indicate the contrary, the equalities and inclusions between measurable sets will be understood except on a set of measure zero. Here and further, $\chi_{S}$ is the characteristic function of a measurable set $S \subset \mathbb{R}^{n}$.

We need the following definition.

Definition 1.2. Let $A: \mathbb{R}^{n} \rightarrow \mathbb{R}^{n}$ be an expansive linear map. $A$ measurable function $f: \mathbb{R}^{n} \rightarrow \mathbb{C}$ is said to be $A$-locally nonzero at a point $\mathbf{x}_{0} \in \mathbb{R}^{n}$ if for any $\varepsilon, r>0$ there exists $j \in \mathbb{N}$ such that

$$
\left|\left\{\mathbf{y} \in A^{-j} B_{r}+\mathbf{x}_{0}: f(\mathbf{y})=0\right\}\right|_{n}<\varepsilon\left|A^{-j} B_{r}\right|_{n} .
$$


Given a measurable set $E \subset \mathbb{R}^{n}$, if the function $f=\chi_{E}$ is $A$-locally nonzero at a point $\mathbf{x}_{0} \in \mathbb{R}^{n}$ we will say that $\mathbf{x}_{0} \in \mathbb{R}^{n}$ is a point of weak $A$-density for the set $E$.

The paper is structured as follows. In Section 2 we will write necessary and sufficient conditions on the scaling functions in a translation invariant $A$-MRA (Theorem 1). Moreover, we will construct a scaling function $\phi$ in a translation invariant $A$-MRA with the property that the support of $\widehat{\phi}$ is bounded and does not contain any open neighborhood of the origin (Theorem 2). Section 3 will be devoted to characterization of low pass filters associated to a scaling function in a translation invariant $A$-MRA (Theorem 3). The proofs of Theorem 1, Theorem 2 and Theorem 3 are given in Sections 4, 5 and 6, respectively.

\section{SCALING FUnCtions}

According to Theorem A, in order to study the scaling functions in a translation invariant $A$-MRA one must study the scaling functions $\phi$ such that $|\widehat{\phi}|=\chi_{S}$ where $S \subset \mathbb{R}^{n}$ is a measurable set.

We prove the following.

Theorem 2.1. Let $A: \mathbb{R}^{n} \rightarrow \mathbb{R}^{n}$ be an expansive linear map such that $A \mathbb{Z}^{n} \subset \mathbb{Z}^{n}$. Let $\phi \in L^{2}\left(\mathbb{R}^{n}\right)$ such that $|\widehat{\phi}|=\chi_{S}$ where $S \subset \mathbb{R}^{n}$ is a measurable set. Then $\phi$ is a scaling function of an A-MRA if and only if the following conditions hold:

1) the origin is a point of weak $A^{*}$-density for the set $S$,

2) $|S|_{n}=1$ and $S_{\mathbb{T}^{n}}=[0,1)^{n}$,

3) $\left(A^{*}\right)^{-1} S \subset S$.

Condition 1) in Theorem 2.1 explains the behavior of the Fourier transform of a scaling function in a neighborhood of the origin. Evidently, if a measurable set contains an open neighborhood of the origin, then the condition 1) in Theorem 2.1 holds. It is well known that there exist measurable sets $S \subset \mathbb{R}$ which do not contain any open neighborhood of the origin and the function $\phi \in L^{2}(\mathbb{R})$ such that $\widehat{\phi}=\chi_{S}$ is a scaling function in a translation invariant multiresolution analysis defined on $L^{2}(\mathbb{R})$ and with the dyadic dilation.

The following example was given by W. R. Madych ([20]). The function $\phi \in L^{2}(\mathbb{R})$ such that $\widehat{\phi}=\chi_{S}$ where

$$
\begin{aligned}
S= & \left(\bigcup_{j=1}^{\infty}\left[-\left(1-2^{-j-1}\right) 2^{-j-1},-2^{-j-1}\right]\right) \cup\left[0, \frac{1}{2}\right] \\
& \cup\left(\bigcup_{j=1}^{\infty}\left[\left(1-\left(1-2^{-j-1}\right) 2^{-j-1}\right),\left(1-2^{-j-1}\right)\right]\right)
\end{aligned}
$$


is a scaling function of a multiresolution analysis.

We prove the following.

TheOREM 2.2. Let $A: \mathbb{R}^{n} \rightarrow \mathbb{R}^{n}$ be an expansive linear map such that $A \mathbb{Z}^{n} \subset \mathbb{Z}^{n}$. There exists a bounded measurable set $S \subset \mathbb{R}^{n}$ such that for any open neighborhood of the origin $U$, there exists an open set included in $S^{c} \cap U$. The function $\phi \in L^{2}\left(\mathbb{R}^{n}\right)$ defined by $\widehat{\phi}=\chi_{S}$ is a scaling function in an $A-M R A$.

\section{LOW PASS FILTERS}

In this section we characterize the measurable functions $H \in L^{2}\left(\mathbb{T}^{n}\right)$ to be a low pass filter in a translation invariant $A$-MRA.

It is well known that for a scaling function in an $A$-MRA there exists an unique low pass filter associated. So, in the proof of Theorem A (see [20]) it is showed that if $H$ is a low pass filter associated to a scaling function $\phi$ such that $|\widehat{\phi}|=\chi_{S}$, then $|H|=\chi_{E}$, where $E \subset \mathbb{R}^{n}$ is a measurable set.

On the other hand, observe that the inverse implication is also true due to if $H \in L^{2}\left(\mathbb{T}^{n}\right)$ such that $|H|=\chi_{E}$ is a low pass filter associated to a scaling function $\phi$ in an $A$-MRA, then $|\widehat{\phi}|=\chi_{S}$ a.e. on $\mathbb{R}^{n}$ where $S=\cap_{j=1}^{\infty}\left(A^{*}\right)^{j} E$. This is a consequence of the fact that if $H \in L^{2}\left(\mathbb{T}^{n}\right)$ is a low pass filter associated to a scaling function $\phi$ in an $A$-MRA, then (see [25])

$$
|\widehat{\phi}(\mathbf{t})|=\prod_{j=1}^{\infty}\left|H\left(\left(A^{*}\right)^{-j} \mathbf{t}\right)\right| \quad \text { a.e. on } \mathbb{R}^{n} .
$$

Let us introduce more notation that we will need in this section.

For a given $\phi \in L^{2}\left(\mathbb{R}^{n}\right)$, set

$$
\Phi_{\phi}(\mathbf{t})=\sum_{\mathbf{k} \in \mathbb{Z}^{n}}|\widehat{\phi}(\mathbf{t}+\mathbf{k})|^{2} .
$$

If $A: \mathbb{R}^{n} \rightarrow \mathbb{R}^{n}$ is an expansive linear map such that $A \mathbb{Z}^{n} \subset \mathbb{Z}^{n}$, then the quotient group $\mathbb{Z}^{n} / A \mathbb{Z}^{n}$ is well defined. We will denote by $\Delta_{A} \subset \mathbb{Z}^{n}$ a full collection of representatives of the cosets of $\mathbb{Z}^{n} / A \mathbb{Z}^{n}$. Recall that there are exactly $d_{A}$ cosets (see [11] and [30, p. 109]). Let us fix $\Delta_{A^{*}}=\left\{\mathbf{p}_{i}\right\}_{i=0}^{d_{A}-1}$ where $\mathbf{p}_{0}=\mathbf{0}$.

Given $H \in L^{\infty}\left(\mathbb{T}^{n}\right)$ the continuous linear operator $\widetilde{P}: L^{1}\left(\mathbb{T}^{n}\right) \rightarrow L^{1}\left(\mathbb{T}^{n}\right)$ defined by

$$
\widetilde{P} f(\mathbf{t})=\sum_{i=0}^{d_{A}-1}\left|H\left(\left(A^{*}\right)^{-1}\left(\mathbf{t}+\mathbf{p}_{i}\right)\right)\right|^{2} f\left(\left(A^{*}\right)^{-1}\left(\mathbf{t}+\mathbf{p}_{i}\right)\right)
$$

is well defined. This operator was first introduced by M. Bownik [2] as a generalization of the analogous operator introduced by W. Lawton [18] for 
dyadic dilations. In the particular case when $|H|=\chi_{E}$, where $E \subset \mathbb{R}^{n}$ is a measurable set, such an operator will be denoted by $P$.

In order not to repeat conditions let us introduce the following class of functions:

$$
\Pi_{A}=\left\{f \in L^{1}\left(\mathbb{T}^{n}\right): f=\chi_{F} \text { where } F \subset \mathbb{R}^{n}\right. \text { is a measurable set }
$$

and the origin is a point of weak $A^{*}$-density for the set $F$ \}.

If $H \in L^{2}\left(\mathbb{T}^{n}\right)$ such that $|H|=\chi_{E}$ is a low pass filter in an $A$-MRA associated to a scaling function $\phi$ such that $|\widehat{\phi}|=\chi_{S}$, then $E=E+\mathbb{Z}^{n}$ and

$$
\chi_{\left(A^{*}\right)^{-1} S}(\mathbf{t})=\chi_{E}(\mathbf{t}) \chi_{S}(\mathbf{t}) \quad \text { a.e. on } \mathbb{R}^{n} .
$$

Further, according to the condition 1) in Theorem 2.1 the origin is a point of weak $A^{*}$-density for the set $S$, then the origin is a point of weak $A^{*}$-density for the set $\left(A^{*}\right)^{-1} S$. Moreover, by the condition 3) in Theorem 2.1, we have $\left(A^{*}\right)^{-1}\left(\left(A^{*}\right)^{-1} S\right) \subset\left(A^{*}\right)^{-1} S$. Therefore, if we call $G=\left(A^{*}\right)^{-1} S$, we conclude that there exists a measurable set $G \subset E$ such that $\left(A^{*}\right)^{-1} G \subset G$ and the origin is a point of weak $A^{*}$-density for the set $G$.

We prove the following result.

Theorem 3.1. Let $A: \mathbb{R}^{n} \rightarrow \mathbb{R}^{n}$ be an expansive linear map such that $A \mathbb{Z}^{n} \subset \mathbb{Z}^{n}$. Let $E \subset \mathbb{R}^{n}, E=E+\mathbb{Z}^{n}$, be a measurable set and there exists a measurable set $G \subset E$ such that $\left(A^{*}\right)^{-1} G \subset G$ and the origin is a point of weak $A^{*}$-density for the set $G$. The following conditions are equivalents.

(I) A function $H \in L^{2}\left(\mathbb{T}^{n}\right)$ such that $|H(\mathbf{t})|=\chi_{E}(\mathbf{t})$ a.e. on $\mathbb{R}^{n}$ is a low pass filter in an $A-M R A$.

(II) (a) $1=\sum_{i=0}^{d_{A}-1} \chi_{E}\left(\mathbf{t}+\left(A^{*}\right)^{-1} \mathbf{p}_{i}\right)$ a.e. on $\mathbb{R}^{n}$.

(b) There exists a measurable set $K \subset \mathbb{R}^{n}$ such that $|K|_{n}=1$ and $\left(A^{*}\right)^{-j} K \subset E, \forall j \geq 1$.

(III) The only function $f \in \Pi_{A}$ invariant under the linear operator $P$ associated to $E$ is the function $f \equiv 1$.

Remark 1. The condition (b) can be replaced by any one of the following conditions:

$\left(\mathrm{b}_{1}\right)$ There exists a measurable set $K \subset \mathbb{R}^{n}$ such that $K_{\mathbb{T}^{n}}=[0,1)^{n}$ and $\left(A^{*}\right)^{-j} K \subset E, \forall j \geq 1$.

( $\left.\mathrm{b}_{2}\right)\left|\bigcap_{j=1}^{\infty}\left(A^{*}\right)^{j} E\right|_{n}=1$.

$\left(\mathrm{b}_{3}\right)\left(\bigcap_{j=1}^{\infty}\left(A^{*}\right)^{j} E\right)_{\mathbb{T}^{n}}=[0,1)^{n}$.

The first step for the study of a function $H \in L^{\infty}\left(\mathbb{T}^{n}\right)$ which is a low pass filter in an $A$-MRA is to assume conditions on the regularity of $H$ at the origin in order to have that the infinite product

$$
\prod_{j=1}^{\infty}\left|H\left(\left(A^{*}\right)^{-j} \mathbf{t}\right)\right|
$$


converges almost everywhere on $\mathbb{R}^{n}$. Assuming regularity at the origin on $H$, previous results on low pass filters can be found in $[2,6,7,9,11,16,18-20,24]$. A first approach to characterize the low pass filters in a translation invariant multiresolution analysis with the dyadic dilation and $n=1$ was presented in [15]. Furthermore, assuming that the infinite product (3.4) converges a.e., characterizations of low pass filters are proved in $[10,13,24,25]$ (see also [26] in a more general context of frame multiresolution analysis).

The condition $(I I I)$ in Theorem 3.1 follows the strategy by Lawton and looks like the conditions in the main results in [25] and in [26]. Here, the main differences are: first, that the set where we look for the fix point for the operator $\widetilde{P}$ defined in (3.3) is not considered previously, in addition, we present a new sufficient condition on $H$ in order to have that the infinite product (3.4) converges a.e.. Such a condition does not involve regularity at the origin on $H$. Finally, the condition $(I I)$ in Theorem 3.1 is of different nature and recalls the conditions given by Cohen for trigonometric polynomial low pass filters.

\section{Proof of Theorem 2.1}

The proof of Theorem 2.1 that we present here is a consequence of the following theorem proved in [5].

TheOREM B. Let $\phi \in L^{2}\left(\mathbb{R}^{n}\right)$. The following conditions are equivalent:

(A) the function $\phi$ is a scaling function of an $A-M R A$;

(B) $\quad(\alpha)$ the function $\widehat{\phi}$ is $A^{*}$-locally nonzero at the origin;

$(\beta)$

$$
\sum_{\mathbf{k} \in \mathbb{Z}^{n}}|\widehat{\phi}(\mathbf{t}+\mathbf{k})|^{2}=1 \quad \text { a.e. on } \mathbb{R}^{n}
$$

$(\gamma)$ there exists a function $H \in L^{\infty}\left(\mathbb{T}^{n}\right)$ such that

$$
\widehat{\phi}(\mathbf{t})=H\left(A^{*-1} \mathbf{t}\right) \widehat{\phi}\left(A^{*-1} \mathbf{t}\right) \quad \text { a.e. on } \mathbb{R}^{n} .
$$

Proof of TheOREM 2.1. If a function $\phi \in L^{2}\left(\mathbb{R}^{n}\right)$ such that $|\widehat{\phi}|=\chi_{S}$ is a scaling function of an $A$-MRA then the condition $(B)$ in Theorem B holds. Thus, the conditions 1), 2) and 3) in Theorem 2.1 follow from the condition $(\alpha),(\beta)$ and $(\gamma)$ respectively.

For a proof of the another implication, we assume that $S \subset \mathbb{R}^{n}$ is a measurable set such that the conditions 1)-3) hold. If we set any function $\phi \in L^{2}\left(\mathbb{R}^{n}\right)$ such that $|\widehat{\phi}|=\chi_{S}$ then the conditions $(\alpha)$ and $(\beta)$ in Theorem B follow from 1) and 2) respectively. Let us check that the condition $(\gamma)$ holds. Since $\sum_{\mathbf{k} \in \mathbb{Z}^{n}} \chi_{S}(\mathbf{t}+\mathbf{k})=1$ a.e. on $\mathbb{R}^{n}$, then $\exists F \subset \mathbb{R}^{n},|F|_{n}=0$, such that $\sum_{\mathbf{k} \in \mathbb{Z}^{n}} \chi_{S}(\mathbf{t}+\mathbf{k})=1$ if $\mathbf{t} \in \mathbb{R}^{n} \backslash F$. Thus the function

$$
H_{0}(\mathbf{t})=\widehat{\phi}\left(A^{*} \mathbf{t}\right)(\widehat{\phi}(\mathbf{t}))^{-1} \quad \text { on } S \backslash F
$$


and the function $H$ defined on the entire space $\mathbb{R}^{n}$ as a $\mathbb{Z}^{n}$-periodic extension of $H_{0}$ and 0 otherwise are well defined.

It is clear that

$$
\widehat{\phi}\left(A^{*} \mathbf{t}\right)=H(\mathbf{t}) \widehat{\phi}(\mathbf{t}) \quad \text { a.e. on } S .
$$

According to condition 3), it remains to prove that

$$
0=H(\mathbf{t}) \widehat{\phi}(\mathbf{t}) \quad \text { a.e. on } \mathbb{R}^{n} \backslash S .
$$

This is a consequence of the condition 2) which means that $\cup_{\mathbf{k} \in \mathbb{Z}^{n}}(S+\mathbf{k})=\mathbb{R}^{n}$ and $|S \cap(S+\mathbf{k})|_{n}=0$ for $\mathbf{k} \in \mathbb{Z}^{n} \backslash\{0\}$. Therefore the proof is finished.

\section{Proof of Theorem 2.2}

We here prove Theorem 2.2 in a constructive way. We first need the following.

LEMma 5.1. Let $\mathcal{A}: \mathbb{R}^{n} \rightarrow \mathbb{R}^{n}$ be a linear invertible map such that $\mathcal{A} \mathbb{Z}^{n} \subset$ $\mathbb{Z}^{n}$ and let $G, J \subset \mathbb{R}^{n}$ be two sets such that $G_{\mathbb{T}^{n}}=J_{\mathbb{T}^{n}}$. Then $(\mathcal{A} G)_{\mathbb{T}^{n}}=$ $(\mathcal{A} J)_{\mathbb{T}^{n}}$.

Proof. By the symmetry in the notation we only prove $(\mathcal{A} G)_{\mathbb{T}^{n}} \subset$ $(\mathcal{A} J)_{\mathbb{T} n}$

Let $\mathbf{y} \in(\mathcal{A} G)_{\mathbb{T}^{n}}$, then there exists $\mathbf{k}_{\mathbf{y}} \in \mathbb{Z}^{n}$ such that $\mathbf{y}+\mathbf{k}_{\mathbf{y}} \in \mathcal{A} G$. Thus, $\mathcal{A}^{-1} \mathbf{y}+\mathcal{A}^{-1} \mathbf{k}_{\mathbf{y}} \in G$. Since $G_{\mathbb{T}^{n}}=J_{\mathbb{T}^{n}}, \exists \mathbf{k}_{\mathbf{y}}^{\prime} \in \mathbb{Z}^{n}$ such that

$$
\mathcal{A}^{-1} \mathbf{y}+\mathcal{A}^{-1} \mathbf{k}_{\mathbf{y}}+\mathbf{k}_{\mathbf{y}}^{\prime} \in J
$$

and so $\mathbf{y}+\mathbf{k}_{\mathbf{y}}+\mathcal{A} \mathbf{k}_{\mathbf{y}}^{\prime} \in \mathcal{A}(J)$.

Finally, by the hypothesis $\mathcal{A} \mathbb{Z}^{n} \subset \mathbb{Z}^{n}$ we know that $\mathbf{k}_{\mathbf{y}}+\mathcal{A} \mathbf{k}_{\mathbf{y}}^{\prime} \in \mathbb{Z}^{n}$ and hence we conclude that $\mathbf{y} \in(\mathcal{A} J)_{\mathbb{T}^{n}}$.

Proof OF THEOREM 2.2. We construct a bounded measurable set $S \subset$ $\mathbb{R}^{n}$ which satisfies the conditions 1)-3) in Theorem 2.1 and also for any open neighborhood of the origin, $U$, there exists an open set included in $S^{c} \cap U$. We make a construction in several steps.

Step 1. Since $A^{*}$ is expansive, $\exists R>0$ such that $\bigcup_{j=0}^{\infty}\left(A^{*}\right)^{-j} B_{R} \subset$ $\left[-\frac{1}{2}, \frac{1}{2}\right)^{n}$. Let $G:=\bigcup_{j=0}^{\infty}\left(A^{*}\right)^{-j} B_{R}$ and observe that the set $G$ can be written by $G=\bigcup_{j=0}^{j_{0}}\left(A^{*}\right)^{-j} B_{R}$ where $j_{0} \in \mathbb{N}$ is such that if $j \geq j_{0}$ then $\left(A^{*}\right)^{-j} B_{R} \subset$ $B_{R}$. Further, the set $G \backslash\left(A^{*}\right)^{-1}(\bar{G})$ is an open measurable set because the set $G$ is an open measurable set and $A^{*}$ is a continuous map. Moreover, $\left|G \backslash\left(A^{*}\right)^{-1}(\bar{G})\right|_{n}>0$. We know that $d_{A}$ is a natural number greater than 1, 
then we have

$$
\begin{aligned}
\left|\left(A^{*}\right)^{-1}(\bar{G})\right|_{n} & =\left|\bigcup_{j=1}^{j_{0}+1}\left(A^{*}\right)^{-j} \overline{B_{R}}\right|_{n}<\sum_{j=1}^{\infty} d_{A}^{-j}\left|B_{R}\right|_{n} \\
& =\frac{1}{d_{A}-1}\left|B_{R}\right|_{n} \leq\left|B_{R}\right|_{n} \leq|G|_{n} .
\end{aligned}
$$

Now, let $G_{l}=\left(A^{*}\right)^{-l} G \backslash\left(A^{*}\right)^{-l-1} G, l \in\{0,1,2, \ldots\}$. Observe that the measurable sets $G_{l}$ are disjoint. Moreover, $G \backslash\{\mathbf{0}\}=\bigcup_{l=0}^{\infty} G_{l}$. If $\mathbf{x} \in G \backslash\{\mathbf{0}\}$, then $\mathbf{x} \in\left(A^{*}\right)^{-j} B_{R}$ for some $j \in\{0,1,2, \ldots\}$. Let $j_{1}$ the biggest number in $\{0,1,2, \ldots\}$ such that $\mathbf{x} \in\left(A^{*}\right)^{-j_{1}} B_{R}$. This $j_{1}$ exists because $A^{*}$ is an expansive linear map. Thus $\mathbf{x} \in G_{j_{1}}=\left(A^{*}\right)^{-j_{1}} G \backslash\left(A^{*}\right)^{-j_{1}-1} G$.

Furthermore, $\exists \mathbf{y}_{\mathbf{0}} \in \mathbb{R}^{n}$ and $\exists r>0$ such that $B_{r}+\mathbf{y}_{\mathbf{0}} \subset G_{0}$ because $G \backslash\left(A^{*}\right)^{-1} \bar{G} \subset G_{0}$ and $G \backslash\left(A^{*}\right)^{-1} \bar{G}$ is an open measurable set of positive measure.

Step 2. Let

$$
S_{0}=\bigcup_{j=0}^{\infty}\left(G_{j} \backslash\left(A^{*}\right)^{-j}\left(B_{2^{-j} r}+\mathbf{y}_{\mathbf{0}}\right)\right) .
$$

The bounded measurable set $S_{0}$ satisfies the following properties:

i) $\left|S_{0}\right|_{n}>0$;

ii) $\left|S_{0}\right|_{n}=\left|\left(S_{0}\right)_{\mathbb{T}^{n}}\right|_{n}$;

iii) $\left(A^{*}\right)^{-1} S_{0} \subset S_{0}$;

$i v)$ the origin is a point of weak $A^{*}$-density for the set $S_{0}$;

$v$ ) the set $S_{0}$ has a nonempty interior.

The properties $i$ ) and $v$ ) hold because $\left(A^{*}\right)^{-j}\left(B_{r}+\mathbf{y}_{\mathbf{0}}\right) \subset G_{j}$. The property $i$ i) follows from $S_{0} \subset\left[-\frac{1}{2}, \frac{1}{2}\right)^{n}$. The property $\left.i i i\right)$ is satisfied because

$$
\left(A^{*}\right)^{-1} S_{0} \subset \bigcup_{j=1}^{\infty}\left(G_{j} \backslash\left(A^{*}\right)^{-j}\left(B_{2^{-j} r}+\mathbf{y}_{\mathbf{0}}\right)\right) \subset S_{0} .
$$

Let us see that the property $i v$ ) holds. Given $l \in \mathbb{N}$,

$$
\begin{aligned}
\frac{\left|\left(A^{*}\right)^{-l} G \bigcap\left(S_{0}\right)^{c}\right|_{n}}{\left|\left(A^{*}\right)^{-l} G\right|_{n}} & =\frac{\left|\left(\bigcup_{j=l}^{\infty} G_{j}\right) \bigcap\left(\bigcup_{j=0}^{\infty}\left(G_{j} \backslash\left(A^{*}\right)^{-j}\left(B_{2^{-j} r}+\mathbf{y}_{\mathbf{0}}\right)\right)\right)^{c}\right|_{n}}{\left|\left(A^{*}\right)^{-l} G\right|_{n}} \\
& =\frac{\left|\bigcup_{j=l}^{\infty}\left(A^{*}\right)^{-j}\left(B_{2^{-j} r}+\mathbf{y}_{0}\right)\right|_{n}}{\left|\left(A^{*}\right)^{-l} G\right|_{n}} \\
& =\frac{\left|B_{r}\right|_{n}}{|G|_{n}} 2^{-l n} \sum_{j=0}^{\infty}\left(2^{n} d_{A}\right)^{-j},
\end{aligned}
$$

where the second and third equalities are true because the sets $G_{j}$ are disjoint and $\left(A^{*}\right)^{-j}\left(B_{2^{-j} r}+\mathbf{y}_{\mathbf{0}}\right) \subset G_{j}$. Observe that $\sum_{j=0}^{\infty}\left(2^{n} d_{A}\right)^{-j}$ converges because 
$d_{A}>1$. Thus, we have

$$
\lim _{l \rightarrow \infty} \frac{\left|\left(A^{*}\right)^{-l} G \bigcap\left(S_{0}\right)^{c}\right|_{n}}{\left|A^{-l} G\right|_{n}}=0 .
$$

So, the origin is a point of weak $A^{*}$-density for the set $S_{0}$ follows.

Step 3. We construct a bounded measurable set $S_{1} \subset \mathbb{R}^{n}$ from the set $S_{0}$. We take the bounded measurable set $\widetilde{F}=A^{*} S_{0} \backslash \cup_{\mathbf{k} \in \mathbb{Z}^{n}}\left(S_{0}+\mathbf{k}\right)$. Given $\mathbf{k} \in \mathbb{Z}^{n}$ we denote by $Q_{\mathbf{k}}=[0,1)^{n}+\mathbf{k}$ and also let us fix $\Omega: \mathbb{N} \rightarrow \mathbb{Z}^{n}$ an one by one and onto application such that if $j_{1}<j_{2}$ then $\left\|\Omega\left(j_{1}\right)\right\| \leq\left\|\Omega\left(j_{2}\right)\right\|$.

For every $j \in \mathbb{N}$ we take the following measurable sets

$$
\widetilde{F}_{j}=\left(\widetilde{F} \cap Q_{\Omega(j)}\right)-\Omega(j) \quad \text { and } \quad F_{j}=\left(\widetilde{F}_{j} \backslash \cup_{\substack{l \in \mathbb{N} \\ l<j}} \widetilde{F}_{l}\right)+\Omega(j) .
$$

Then $F=\cup_{j \in \mathbb{N}} F_{j}$ is a bounded measurable set. It satisfies

$$
F_{\mathbb{T}^{n}}=\left(A^{*} S_{0}\right)_{\mathbb{T}^{n}} \backslash\left(S_{0}\right)_{\mathbb{T}^{n}}
$$

and

$$
\left|\left(F+\mathbf{k}_{1}\right) \cap\left(F+\mathbf{k}_{2}\right)\right|_{n}=0 \quad \text { if } \quad \mathbf{k}_{1}, \mathbf{k}_{2} \in \mathbb{Z}^{n}, \mathbf{k}_{1} \neq \mathbf{k}_{2} .
$$

Now, we take the bounded measurable set $S_{1}=F \cup S_{0}$. Observe that if $\left|A^{*} S_{0} \backslash\left(S_{0}+\mathbb{Z}^{n}\right)\right|_{n}=0$, then $S_{1}=S_{0}$.

The set $S_{1}$ satisfies the following properties:

$\left.i_{1}\right)\left(S_{1}\right)_{\mathbb{T}^{n}}=\left(A^{*} S_{0}\right)_{\mathbb{T}^{n}}$

ii $)\left|S_{1}\right|_{n}=\left|\left(S_{1}\right)_{\mathbb{T}^{n}}\right|_{n}$;

iii $\left.i_{1}\right)\left(A^{*}\right)^{-1} S_{1} \subset S_{1}$;

$\left.i v_{1}\right)$ the origin is a point of weak $A^{*}$-density for the set $S_{1}$.

By (5.2) and the condition $i i i)$ in Step 2, we have

$$
\begin{aligned}
& \left(S_{1}\right)_{\mathbb{T}^{n}}=\left(F \cup S_{0}\right)_{\mathbb{T}^{n}}=(F)_{\mathbb{T}^{n}} \cup\left(S_{0}\right)_{\mathbb{T}^{n}} \\
= & \left(\left(A^{*} S_{0}\right)_{\mathbb{T}^{n}} \backslash\left(S_{0}\right)_{\mathbb{T}^{n}}\right) \cup\left(S_{0}\right)_{\mathbb{T}^{n}}=\left(A^{*} S_{0}\right)_{\mathbb{T}^{n} .}
\end{aligned}
$$

Thus, the property $i_{1}$ ) holds.

We see that the set $S_{1}$ satisfies the property $i i_{1}$ ). By the definition of the set $S_{1}$,

$$
\left|S_{1}\right|_{n}=\left|F \cup S_{0}\right|_{n}=|F|_{n}+\left|S_{0}\right|_{n},
$$

where the last equality holds because $F$ and $S_{0}$ are disjoint measurable sets. By the condition (5.3) we have $|F|_{n}=\left|(F)_{\mathbb{T}^{n}}\right|_{n}$, and also, we know that $\left|S_{0}\right|_{n}=\left|\left(S_{0}\right)_{\mathbb{T}^{n}}\right|_{n}$. Thus

$$
\begin{aligned}
\left|S_{1}\right|_{n} & =\left|(F)_{\mathbb{T}^{n}}\right|_{n}+\left|\left(S_{0}\right)_{\mathbb{T}^{n}}\right|_{n}=\left|(F)_{\mathbb{T}^{n}} \cup\left(S_{0}\right)_{\mathbb{T}^{n}}\right|_{n} \\
& =\left|\left(F \cup S_{0}\right)_{\mathbb{T}^{n}}\right|_{n}=\left|\left(S_{1}\right)_{\mathbb{T}^{n}}\right|_{n},
\end{aligned}
$$

where the second equality is true because $\left|(F)_{\mathbb{T}^{n}} \bigcap\left(S_{0}\right)_{\mathbb{T}^{n}}\right|_{n}=0$.

According to the condition $i$ ii) in Step 2, we have

$$
\left(A^{*}\right)^{-1} S_{1} \subset\left(A^{*}\right)^{-1}\left[\left(A^{*} S_{0} \backslash S_{0}\right) \cup S_{0}\right] \subset S_{0} \subset S_{1},
$$


that it is the property $\left.i i_{1}\right)$.

The set $S_{1}$ satisfies the condition $i v_{1}$ ) because the origin is a point of weak $A^{*}$-density for the set $S_{0}$ and $S_{0} \subset S_{1}$.

Step 4. Iterating the procedure in Step 3 , for every $N \in\{1,2, \ldots\}$ we build a bounded measurable set $S_{N} \subset \mathbb{R}^{n}$ which satisfies the following properties:

$\left.i_{N}\right)\left(S_{N}\right)_{\mathbb{T}^{n}}=\left(\left(A^{*}\right)^{N} S_{0}\right)_{\mathbb{T}^{n}} ;$

$\left.i i_{N}\right)\left|S_{N}\right|_{n}=\left|\left(S_{N}\right)_{\mathbb{T}^{n}}\right|_{n} ;$

$\left.i i i_{N}\right)\left(A^{*}\right)^{-1} S_{N} \subset S_{N}$;

$\left.i v_{N}\right)$ the origin is a point of weak $A^{*}$-density for the set $S_{N}$.

In order to see that the set $S_{N}$ satisfies conditions $\left.\left.\left.i_{N}\right), i i_{N}\right), i i i_{N}\right)$ and $\left.i v_{N}\right)$, we proceed by induction with respect to $N$.

We have just proved that the sets $S_{1}$ satisfies the conditions $i_{1}$ ), $i i_{1}$ ), $\left.i i i_{1}\right)$ and $\left.i v_{1}\right)$ in Step 3. Let $N \geq 1$ and we assume that $S_{N}$ satisfies all the conditions $\left.\left.\left.i_{N}\right), i i_{N}\right), i i i_{N}\right)$ and $\left.i v_{N}\right)$.

Using the hypothesis of induction $\left.i i_{N}\right), i i i_{N}$ ) and $\left.i v_{N}\right)$, the conditions $\left.\left.i i_{N+1}\right), i i i_{N+1}\right)$ and $i v_{N+1}$ ) can be proved in a similar way as the proof done in Step 3 where we have proved that the set $S_{1}$ satisfies the conditions $i i_{1}$ ), $\left.i i i_{1}\right)$ and $i v_{1}$ ).

We show that $S_{N+1}$ satisfies $\left.i_{N+1}\right)$. From the hypothesis of induction $\left.i i i_{N}\right)$ and in a similar way as (5.4) we get

$$
\left(S_{N+1}\right)_{\mathbb{T}^{n}}=\left(A^{*} S_{N}\right)_{\mathbb{T}^{n}}
$$

According to the hypothesis of induction $i_{N}$ ) and Lemma 5.1, we conclude that

$$
\left(S_{N+1}\right)_{\mathbb{T}^{n}}=\left(\left(A^{*}\right)^{N+1} S_{0}\right)_{\mathbb{T}^{n}} .
$$

Step 5. Let $B \subset S_{0}$ be an open ball. This open ball exists according to the condition $v$ ) in Step 2. Then there exists $N_{0} \in\{1,2, \ldots\}$ such that $\left(\left(A^{*}\right)^{N_{0}} B\right)_{\mathbb{T}^{n}}=[0,1)^{n}$ because $A^{*}$ is an expansive linear map.

We denote by $S$ the corresponding bounded measurable set $S_{N_{0}}$. First of all, we assert that the set $S$ satisfies the conditions 1)-3) in Theorem 2.1. The set $S$ satisfies those conditions 1) and 3) according to the conditions $i v_{N_{0}}$ ) and $i i i_{N_{0}}$ ) in Step 4 respectively. Furthermore, by the condition $\left.i_{N_{0}}\right)$ in Step 4 we have

$$
S_{\mathbb{T}^{n}}=\left(\left(A^{*}\right)^{N_{0}} S_{0}\right)_{\mathbb{T}^{n}} \supset\left(\left(A^{*}\right)^{N_{0}} B\right)_{\mathbb{T}^{n}}=[0,1)^{n},
$$

and also, by the condition $\left.i i_{N_{0}}\right)$ in Step 4 ,

$$
|S|_{n}=\left|\left(S_{N_{0}}\right)_{\mathbb{T}^{n}}\right|_{n}=1 .
$$

Therefore, the set $S$ satisfies the condition 2).

Finally, that given $U \subset \mathbb{R}^{n}$ an open neighborhood of the origin there exists an open set included in $S^{c} \cap U$ is a consequence of the facts that $S \subset A^{* N_{0}} S_{0}$ and the set $S_{0}$ can be written by $S_{0}=\left(\bigcup_{j=0}^{\infty}\left(G_{j}\right)\right) \backslash\left(\bigcup_{j=0}^{\infty}\left(A^{*}\right)^{-j}\left(B_{2^{-j} r}+\mathbf{y}_{\mathbf{0}}\right)\right)$ 
because the sets $G_{j}, j=0,1,2, \ldots$, are disjoint and $\left(A^{*}\right)^{-j}\left(B_{2^{-j} r}+\mathbf{y}_{\mathbf{0}}\right) \subset G_{j}$. This finishes the proof.

\section{Proof of Theorem 3.1}

In the proof of Theorem 3.1 we need the following properties and auxiliary results on low pass filters and locally nonzero measurable functions.

Different versions of the following proposition appear in several publications (cf. $[2,9,16,21,22]$ ).

Proposition A. Let $H$ be a low pass filter associated to a scaling function of an $A-M R A$. Then

$$
\sum_{i=0}^{d_{A}-1}\left|H\left(\mathbf{t}+\left(A^{*}\right)^{-1} \mathbf{p}_{i}\right)\right|^{2}=1 \quad \text { a.e. on } \mathbb{R}^{n} \text {. }
$$

Proposition B. Let $H \in L^{\infty}\left(\mathbb{T}^{n}\right)$ be a function such that (6.1) holds. If the infinite product $\prod_{j=1}^{\infty}\left|H\left(\left(A^{*}\right)^{-j} \mathbf{t}\right)\right|$ converges almost everywhere on $\mathbb{R}^{n}$ then

a) the function $\widehat{\theta}(\mathbf{t}):=\prod_{j=1}^{\infty}\left|H\left(\left(A^{*}\right)^{-j} \mathbf{t}\right)\right|$ belongs to $L^{2}\left(\mathbb{R}^{n}\right)$ and $\|$ $\widehat{\theta} \|_{L^{2}\left(\mathbb{R}^{n}\right)} \leq 1$,

b) $\Phi_{\theta}(\mathbf{t}) \leq 1$ a.e. on $\mathbb{R}^{n}$,

c) $\Phi_{\theta}$ is a fixed point for the operator $\widetilde{P}$,

where the function $\theta$ is defined by $\widehat{\theta}(\mathbf{t})=\prod_{j=1}^{\infty}\left|H\left(\left(A^{*}\right)^{-j} \mathbf{t}\right)\right|$.

In the above proposition, the condition a) was proved by M. Bownik ([2], see also $[9,16])$, the condition b) was proved in the proof of main result in $[25]$ and the condition c) was also proved in [2].

The following lemma was proved in [5]. Note that the equality (ii) does not appear in the original result but it is a direct consequence of the proof of the condition $(i)$.

Lemma A. Let $g \in L^{2}\left(\mathbb{T}^{n}\right)$, let $\mathcal{A}: \mathbb{R}^{n} \rightarrow \mathbb{R}^{n}$ be a linear invertible map such that $\mathcal{A} \mathbb{Z}^{n} \subset \mathbb{Z}^{n}$ and let $\hat{\mathcal{A}}: \mathbb{T}^{n} \rightarrow \mathbb{T}^{n}$ be the induced endomorphism. Then

(i) $\int_{\mathbb{T}^{n}} g(\hat{\mathcal{A}} \mathbf{t}) d \mathbf{t}=\int_{\mathbb{T}^{n}} g(\mathbf{t}) d \mathbf{t}$.

(ii) $\int_{[0,1]^{n}} g(\mathbf{t}) d \mathbf{t}=d_{\mathcal{A}}^{-1} \int_{[0,1]^{n}} \sum_{i=0}^{d_{\mathcal{A}}-1} g\left(\mathcal{A}^{-1} \mathbf{t}+\mathcal{A}^{-1} \mathbf{q}_{i}\right) d \mathbf{t}$,

where $\left\{\mathbf{q}_{i}\right\}_{i=1}^{d_{\mathcal{A}}-1} \subset \mathbb{Z}^{n}$ is a full collection of representatives of $\mathbb{Z}^{n} / \mathcal{A} \mathbb{Z}^{n}$.

In [25] the following remark was proved.

REMARK A. A $\mathbb{Z}^{n}$-periodic measurable function $H$ is a low pass filter of an $A-M R A$ if and only if $|H|$ is a low pass filter of some A-MRA.

The following proposition on locally nonzero functions was proved in [25]. 
Proposition C. Let $A: \mathbb{R}^{n} \rightarrow \mathbb{R}^{n}$ be an expansive linear invertible map. Let $f: \mathbb{R}^{n} \longrightarrow \mathbb{C}$ be a measurable function that is $A$-locally nonzero at the origin. Then, there exists $\left\{j_{k}\right\}_{k=1}^{\infty} \subset \mathbb{N}, j_{k+1}>j_{k}$, such that for a.e. $\mathbf{x}$ in $\mathbb{R}^{n}$ there is $k_{0} \in \mathbb{N}$ such that if $k \geq k_{0}$, then $f\left(A^{-j_{k}} \mathbf{x}\right) \neq 0$.

We present a condition in order to the infinity product $\prod_{j=1}^{\infty} \chi_{E}\left(\left(A^{*}\right)^{-j} \mathbf{t}\right)$ converges a.e.

Lemma 6.1. Let $A: \mathbb{R}^{n} \rightarrow \mathbb{R}^{n}$ be an expansive linear map. Let $E \subset \mathbb{R}^{n}$, $E=E+\mathbb{Z}^{n}$, be a measurable set such that $\chi_{E}$ satisfies (6.1). Moreover, there exists a measurable set $G \subset E$ such that $\left(A^{*}\right)^{-1} G \subset G$ and the origin is a point of weak $A^{*}$-density for the set $G$. Then the infinite product $\prod_{j=1}^{\infty} \chi_{E}\left(\left(A^{*}\right)^{-j} \mathbf{t}\right)$ converges a.e. to the function $\chi_{S}$ where $S=\cap_{j=1}^{\infty}\left(A^{*}\right)^{j} E$. In addition, $|S|_{n} \leq$ 1 and the origin is a point of weak $A^{*}$-density for the set $S$.

ProOF. First of all, we prove that the infinite product $\prod_{j=1}^{\infty} \chi_{E}\left(\left(A^{*}\right)^{-j} \mathbf{t}\right)$ converges a.e. on $\mathbb{R}^{n}$. According to Proposition $\mathrm{C}$ there exists a strictly increasing sequence $\left\{j_{k}\right\}_{k=1}^{\infty} \subset \mathbb{N}$ such that for almost every point $\mathbf{t} \in \mathbb{R}^{n}$

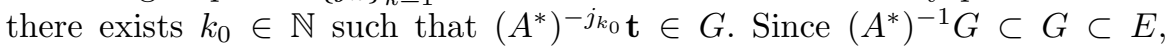
if $L \geq j_{k_{0}}$ then $\prod_{j=j_{k_{0}}}^{L} \chi_{E}\left(\left(A^{*}\right)^{-j} \mathbf{t}\right)=1$ a.e. Letting $L \rightarrow \infty$, we obtain $\prod_{j=j_{k_{0}}}^{\infty} \chi_{E}\left(\left(A^{*}\right)^{-j} \mathbf{t}\right)=1$ a.e. Therefore, $\prod_{j=1}^{\infty} \chi_{E}\left(\left(A^{*}\right)^{-j} \mathbf{t}\right)$ converges a.e. on $\mathbb{R}^{n}$. Furthermore, we show that $\prod_{j=1}^{\infty} \chi_{E}\left(\left(A^{*}\right)^{-j} \mathbf{t}\right)$ converges a.e. to $\chi_{S}$. For almost every point $\mathbf{t} \in S$, we have $\prod_{j=1}^{\infty} \chi_{E}\left(\left(A^{*}\right)^{-j} \mathbf{t}\right)=1$. If $\mathbf{t} \in \mathbb{R}^{n} \backslash S$, then there exists $j_{0} \in \mathbb{N}$ such that $\mathbf{t} \notin\left(A^{*}\right)^{j_{0}} E$, thus $\chi_{E}\left(\left(A^{*}\right)^{-j_{0}} \mathbf{t}\right) \prod_{\substack{j=1 \\ j \neq j_{0}}}^{\infty} \chi_{E}\left(\left(A^{*}\right)^{-j} \mathbf{t}\right)=0$.

That $|S|_{n} \leq 1$ follows from the condition a) in Proposition B.

Finally, since the set $G \subset E$ satisfies that $\left(A^{*}\right)^{-1} G \subset G$, then $G \subset S$. Moreover, since the origin is a point of weak $A^{*}$-density for the set $G$, we conclude that the origin is a point of weak $A^{*}$-density for the set $S$. Therefore, the proof is finished.

Proof of TheOREM 3.1. Let us begin with the proof of the implication $(I) \Longrightarrow(I I I)$. That $f \equiv 1$ is invariant under $P$ is an immediate consequence of Proposition A.

Suppose that $f \in \Pi_{A}$ is a fixed point of the operator $P$. We will show that

$$
\int_{[0,1]^{n}} f(\mathbf{t}) d \mathbf{t} \geq 1
$$

This condition together with $f \in \Pi_{A}$ will show that $f(\mathbf{t})=1$ a.e. on $\mathbb{R}^{n}$. 
Using the equality $P f=f$, we obtain

$$
\begin{aligned}
\int_{[0,1]^{n}} f(\mathbf{t}) d \mathbf{t} & =\int_{[0,1]^{n}} P(f)(\mathbf{t}) d \mathbf{t} \\
& =\int_{[0,1]^{n}} \sum_{i=0}^{d_{A}-1} \chi_{E}\left(\left(A^{*}\right)^{-1}\left(\mathbf{t}+\mathbf{p}_{i}\right)\right) f\left(\left(A^{*}\right)^{-1}\left(\mathbf{t}+\mathbf{p}_{i}\right)\right) d \mathbf{t} \\
& =d_{A} \int_{[0,1]^{n}} \chi_{E}(\mathbf{t}) f(\mathbf{t}) d \mathbf{t}=d_{A} \int_{\left[-\frac{1}{2}, \frac{1}{2}\right]^{n}} \chi_{E}(\mathbf{t}) f(\mathbf{t}) d \mathbf{t},
\end{aligned}
$$

where the third equality follows from the condition (ii) of Lemma A.

Putting $A^{*} \mathbf{t}=\mathbf{v}$, we obtain

$$
\begin{aligned}
\int_{[0,1]^{n}} f(\mathbf{t}) d \mathbf{t} & =\int_{\mathbb{R}^{n}} \chi_{E}\left(\left(A^{*}\right)^{-1} \mathbf{v}\right) f\left(\left(A^{*}\right)^{-1} \mathbf{v}\right) \chi_{\left[-\frac{1}{2}, \frac{1}{2}\right]^{n}}\left(\left(A^{*}\right)^{-1} \mathbf{v}\right) d \mathbf{v} \\
& =\int_{\mathbb{R}^{n}} \chi_{E}\left(\left(A^{*}\right)^{-1} \mathbf{t}\right) P(f)\left(\left(A^{*}\right)^{-1} \mathbf{t}\right) \chi_{\left[-\frac{1}{2}, \frac{1}{2}\right]^{n}}\left(\left(A^{*}\right)^{-1} \mathbf{t}\right) d \mathbf{t},
\end{aligned}
$$

since $P f=f$.

Iterating the above computations and using the condition $A^{*} \mathbb{Z}^{n} \subset \mathbb{Z}^{n}$, we obtain

$$
\int_{[0,1]^{n}} f(\mathbf{t}) d \mathbf{t}=\int_{\mathbb{R}^{n}} \prod_{j=1}^{N} \chi_{E}\left(\left(A^{*}\right)^{-j} \mathbf{t}\right) f\left(\left(A^{*}\right)^{-N} \mathbf{t}\right) \chi_{\left[-\frac{1}{2}, \frac{1}{2}\right]^{n}}\left(\left(A^{*}\right)^{-N} \mathbf{t}\right) d \mathbf{t} .
$$

Let

$$
\Gamma_{N} f(\mathbf{t})=\prod_{j=1}^{N} \chi_{E}\left(\left(A^{*}\right)^{-j} \mathbf{t}\right) f\left(\left(A^{*}\right)^{-N} \mathbf{t}\right) \chi_{\left[-\frac{1}{2}, \frac{1}{2}\right]^{n}}\left(\left(A^{*}\right)^{-N} \mathbf{t}\right), \quad \text { for } N \in \mathbb{N} .
$$

Since $f \in \Pi_{A}$, according to Proposition C there exists a strictly increasing sequence of natural numbers $\left\{l_{N}\right\}_{N=1}^{\infty} \subset \mathbb{N}$, such that for a.e. $\mathbf{t}$ in $\mathbb{R}^{n}$ there exists $N_{0} \in \mathbb{N}$ such that if $N \geq N_{0}$, then $\chi_{\left[-\frac{1}{2}, \frac{1}{2}\right]^{n}}\left(\left(A^{*}\right)^{-l_{N}} \mathbf{t}\right) f\left(\left(A^{*}\right)^{-l_{N}} \mathbf{t}\right)=1$. In addition, according to Lemma 6.1 the infinite product $\prod_{j=1}^{\infty} \chi_{E}\left(\left(A^{*}\right)^{-j} \mathbf{t}\right)$ converges a.e. on $\mathbb{R}^{n}$. Hence

$$
\lim _{N \longrightarrow \infty} \Gamma_{l_{N}} f(\mathbf{t})=\prod_{j=1}^{\infty} \chi_{E}\left(\left(A^{*}\right)^{-j} \mathbf{t}\right), \quad \text { a.e. on } \mathbb{R}^{n} .
$$

By Fatou's lemma and (6.2)

$$
\begin{aligned}
\int_{[0,1]^{n}} f(\mathbf{t}) d \mathbf{t} & =\lim _{N \rightarrow \infty} \int_{\mathbb{R}^{n}} \Gamma_{l_{N}} f(\mathbf{t}) d \mathbf{t} \geq \int_{\mathbb{R}^{n}} \lim _{N \rightarrow \infty} \Gamma_{l_{N}} f(\mathbf{t}) d \mathbf{t} \\
& =\int_{\mathbb{R}^{n}} \prod_{j=1}^{\infty} \chi_{E}\left(\left(A^{*}\right)^{-j} \mathbf{t}\right) d \mathbf{t}=1,
\end{aligned}
$$


where the last equality holds according to the condition $(I)$ and the equality (3.1).

Let us prove $(I I I) \Longrightarrow(I I)$. The condition (a) in (II) follows immediately from the condition $(I I I)$. To prove the condition (b) in (II), let $K=$ $\cap_{j=1}^{\infty}\left(A^{*}\right)^{j} E$. Thus it remains to verify that $|K|_{n}=1$.

According to Lemma $6.1,|K|_{n} \leq 1$. Thus there exists $\theta \in L^{2}\left(\mathbb{R}^{n}\right)$ a measurable function defined by $\widehat{\theta}=\chi_{K}$. The condition c) in Proposition B tells us that the $\mathbb{Z}^{n}$-periodic measurable function $\Phi_{\theta}(\mathbf{t})=\sum_{\mathbf{k} \in \mathbb{Z}^{n}} \chi_{K}(\mathbf{t}+\mathbf{k})$ is a fixed point for the operator $P$.

If we prove that $\Phi_{\theta} \in \Pi_{A}$, by the condition $(I I I)$ we will have that $\Phi_{\theta}(\mathbf{t})=1$ a.e. on $\mathbb{T}^{n}$.

By the condition b) in Proposition B

$$
\Phi_{\theta}(\mathbf{t})=\sum_{\mathbf{k} \in \mathbb{Z}^{n}} \chi_{K}(\mathbf{t}+\mathbf{k}) \leq 1 \quad \text { a.e. on } \mathbb{R}^{n} .
$$

Hence $\Phi_{\theta} \in L^{1}\left(\mathbb{T}^{n}\right)$ and also $\Phi_{\theta}=\chi_{F}$ a.e. on $\mathbb{R}^{n}$ where $F \subset \mathbb{R}^{n}$ is a measurable set. In addition, according to Lemma 6.1 the function $\Phi_{\theta}$ is $A^{*}$ locally nonzero at the origin and therefore $\Phi_{\theta} \in \Pi_{A}$.

Further,

$$
|K|_{n}=\int_{\mathbb{R}^{n}} \chi_{K}(\mathbf{t}) d \mathbf{t}=\sum_{\mathbf{k} \in \mathbb{Z}^{n}} \int_{[0,1)^{n}-\mathbf{k}} \chi_{K}(\mathbf{t}) d \mathbf{t}=\int_{[0,1)^{n}} \sum_{\mathbf{k} \in \mathbb{Z}^{n}} \chi_{K}(\mathbf{t}+\mathbf{k}) d \mathbf{t}
$$

and thus we conclude that $|K|_{n}=1$ due to $\Phi_{\theta}(\mathbf{t})=1$ a.e. on $\mathbb{R}^{n}$.

We prove $(I I) \Longrightarrow(I)$. Let $\theta$ be a function such that

$$
\widehat{\theta}(\mathbf{t})=\Pi_{j=1}^{\infty} \chi_{E}\left(\left(A^{*}\right)^{-j} \mathbf{t}\right)=\chi_{S}
$$

where $S=\cap_{j=1}^{\infty}\left(A^{*}\right)^{j} E$. Observe that the function $\theta$ is a well defined measurable function in $L^{2}\left(\mathbb{R}^{n}\right)$ due to Lemma 6.1 . If we prove that the set $S$ satisfies the conditions 1)-3) in Theorem 2.1, then the function $\theta$ is a scaling function in an $A$-MRA. So by the definition of $\widehat{\theta}$, the function $\chi_{E}$ is the low pass filter associated to the scaling function $\theta$.

By Lemma 6.1, the set $S$ satisfies the condition 1). Now, we check that the set $S$ satisfies the condition 2) in Theorem 2.1. By Lemma 6.1, then $|S|_{n} \leq 1$. Further, as the set $K$ in the condition (b) in $(I I)$ is included in $S$ and $|K|_{n}=1$, we have that $|S|_{n}=1$. Moreover, with analogous computations of (6.3) with $S$ instead of the set $K$ and having in mind that $|S|_{n}=1$ and the property b) in Proposition B, we obtain that $\sum_{\mathbf{k} \in \mathbb{Z}^{n}} \chi_{S}(\mathbf{t}+\mathbf{k})=1$ a.e. Hence, $S_{\mathbb{T}^{n}}=[0,1)^{n}$. Obviously the set $S$ satisfies the condition 3) in Theorem 2.1.

We have just prove that the function $\chi_{E}$ is the low pass filter associated to the scaling function $\theta$ in an $A$-MRA. Finally, according to Remark A we finish the proof. 


\section{REFERENCES}

[1] D. Bakić and E. N. Wilson, Scaling sets and orthonormal wavelets with dilations induced by expanding matrices, Glas. Mat. Ser. III 46(66) (2011), 189-213.

[2] M. Bownik, Tight frames of multidimensional wavelets, Dedicated to the memory of Richard J. Duffin. J. Fourier Anal. Appl. 3 (1997), 525-542.

[3] M. Bownik, Z. Rzeszotnik and D. M. Speegle, A characterization of dimension functions of wavelets, Appl. Comput. Harmon. Anal. 10 (2001), 71-92.

[4] C. K. Chui, An introduction to wavelets, Academic Press, Inc., Boston, 1992.

[5] P. Cifuentes, K. S. Kazarian and A. San Antolín, Characterization of scaling functions in a multiresolution analysis, Proc. Amer. Math. Soc. 133 (2005), 1013-1023.

[6] A. Cohen, Ondelettes, analyses multirésolutions et filtres miroirs en quadrature, Ann. Inst. H. Poincaré Anal. Non Linéaire 7 (1990), 439-459.

[7] A. Cohen, I. Daubechies and J. C. Feauveau, Biorthogonal bases of compactly supported wavelets, Comm. Pure Appl. Math. 45 (1992), 485-560.

[8] X. Dai and D. R. Larson, Wandering vectors for unitary systems and orthogonal wavelets, Mem. Amer. Math. Soc., 134 (1998), no. 640, pp. 68.

[9] I. Daubechies, Ten lectures on wavelets, SIAM, Philadelphia, 1992.

[10] V. Dobrić, R. F. Gundy and P. Hitczenko, Characterizations of orthonormal scale functions: a probabilistic approach, J. Geom. Anal. 10 (2000), 417-434.

[11] K. Gröchenig and W. R. Madych, Multiresolution analysis, Haar bases, and selfsimilar tilings of $R^{n}$, IEEE Trans. Inform. Theory 38 (1992), 556-568.

[12] Q. Gu and D. Han, On multiresolution analysis (MRA) wavelets in $\mathbf{R}^{n}$, J. Fourier Anal. Appl. 6 (2000), 437-447.

[13] R. F. Gundy, Low-pass filters, martingales, and multiresolution analyses, Appl. Comput. Harmon. Anal. 9 (2000), 204-219.

[14] Y-H. Ha, H. Kang, J. Lee and J. K. Seo, Unimodular wavelets for $L^{2}$ and the Hardy space $H^{2}$, Michigan Math. J. 41 (1994), 345-361.

[15] E. Hernández, X. Wang and G. Weiss, Smoothing minimally supported frequency wavelets. II. J. Fourier Anal. Appl. 3 (1997), 23-41.

[16] E. Hernández and G. Weiss, A first course on wavelets, CRC Press, Inc. 1996.

[17] K. S. Kazarian and A. San Antolin, Characterization of scaling functions in a frame multiresolution analysis in $\mathrm{H}_{G}^{2}$, in: Topics in mathematical analysis and applications, World Sci. Publ., Hackensack, 2008, 118-140.

[18] W. M. Lawton, Necessary and sufficient conditions for constructing orthonormal wavelet bases, J. Math. Phys. 32 (1991), 57-61.

[19] W. M. Lawton, Tight frames of compactly supported affine wavelets, J. Math. Phys. 31 (1990), 1898-1901.

[20] W. R. Madych, Some elementary properties of multiresolution analyses of $L^{2}\left(R^{d}\right)$, in: Wavelets, Ch. Chui ed., Academic Press, Boston, 1992, 259-294.

[21] S. Mallat, Multiresolution approximations and wavelet orthonormal bases of $L^{2}(\mathbf{R})$, Trans. Amer. Math. Soc. 315 (1989), 69-87.

[22] Y. Meyer, Ondelettes et opérateurs. I, (French) Hermann, Paris, 1990 [English Translation: Wavelets and operators. I, Cambridge University Press, 1992.]

[23] M. Papadakis, Unitary mappings between multiresolution analysis of $L^{2}(\mathbf{R})$ and a parameterization of low-pass filters, J. Fourier Anal. Appl. 4 (1998), 199-214.

[24] M. Papadakis, H. Šikić and G. Weiss, The characterization of low pass filters and some basic properties of wavelets, scaling functions and related concepts, J. Fourier Anal. Appl. 5 (1999), 495-521.

[25] A. San Antolín, Characterization of low pass filters in a multiresolution analysis, Studia Math. 190 (2009), 99-116. 
[26] A. San Antolín, On low pass filters in a frame multiresolution analysis, Tohoku Math. J. (2) 63 (2011), 427-439.

[27] M. Soto-Bajo, Closure of dilates of shift-invariant subspaces, Cent. Eur. J. Math. 11 (2013), 1785-1799.

[28] R. S. Strichartz, Construction of orthonormal wavelets, in: Wavelets: mathematics and applications, CRC, Boca Raton, 1994, 23-50.

[29] E. Weber, On the translation invariance of wavelet subspaces, J. Fourier Anal. Appl. 6 (2000), 551-558.

[30] P. Wojtaszczyk, A mathematical introduction to wavelets, Cambridge University Press, Cambridge, 1997.

A. San Antolín

Departamento de Análisis Matemático

Universidad de Alicante

03080 Alicante

Spain

E-mail: angel.sanantolin@ua.es

Received: 17.6.2013.

Revised: 5.10.2013. 